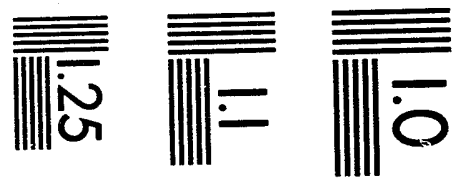

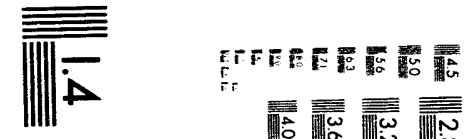

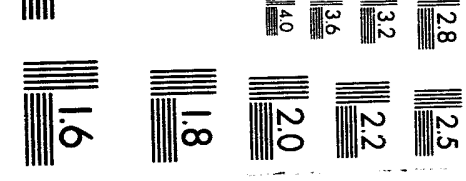



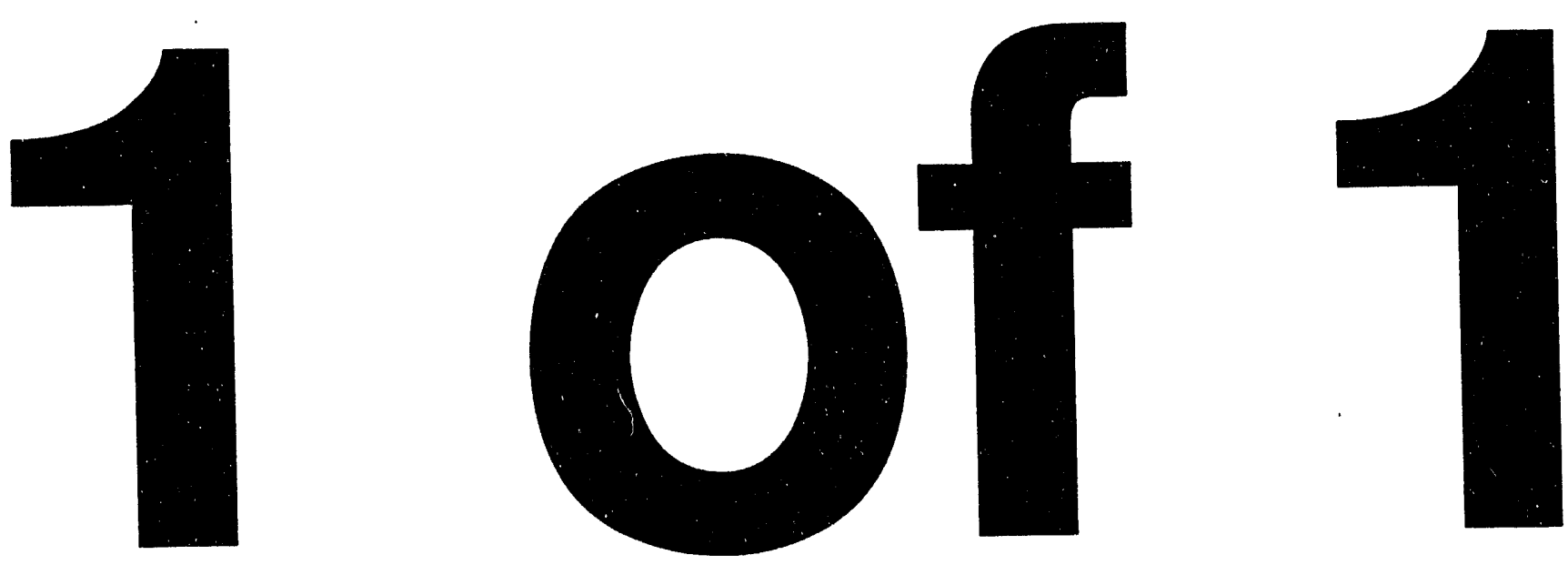


\section{Comparisons of Gyrofluid and Gyrokinetic Simulations}

S. E. Parker, W. Dorland*, R. A. Santoro, M. A. Beer, Q. P. Liu, W. W. Lee, and G. W. Hammett

Princeton Plasma Physics Laboratory, Princeton University, P.O. Box 451, Princeton NJ 08543

*Present address: IFS, The University of Texas, Austin, TX, 78712 


\begin{abstract}
The gyrokinetic and gyrofluid models show the most promise for large scale simulations of tokamak microturbulence. This paper discusses detailed comparisons of these two complementary approaches. Past comparisons with linear theory have been fairly good, therefore the emphasis here is on nonlinear comparisons. Simulations include simple two dimensional slab test cases, turbulent three dimensional slab cases, and toroidal cases, each modeling the nonlinear evolution of the ion temperature gradient instability. There is good agreement in both turbulent and coherent nonlinear slab comparisons in terms of the ion heat flux, both in magnitude and scaling with magnetic shear. However, the nonlinear saturation level for $|\Phi|$ in the slab comparisons show differences of approximately $40 \%$. Preliminary toroidal comparisons show agreement within $50 \%$, in terms of ion heat flux and saturation level.
\end{abstract}




\section{Introduction}

Successful work over the past decade on the formulation of gyrokinetic equations ${ }^{1-3}$ and the more recent development of the gyrofluid equations ${ }^{4-6}$ provide two practical and complementary approaches for simulating low frequency gradient driven microturbulence in tokamak plasmas. The development of these model equations along with the astounding advances being made in high performance computing now make large scale numerical simulations an important tool for the improved unders anding of anomalous transport. These advances have motivated a collaborative effort witnin the magnetic fusion community termed the "Numerical Tokamak Project" (NTP) utilizing these new computational tools. ${ }^{7}$ Though still in its early stages, the NTP has generated interesting physics results, including the observation that the turbulent spectrum peaks at significantly longer wavelengths than the linearly most unstable $\operatorname{modes}^{8-10}$ along with results showing very similar spectral features as recent experimental core fluctuation measurements. ${ }^{8,11,12}$ In addition, important self-generated poloidal shear flows have been observed in core Ion Temperature Gradient (ITG) mode simulations, ${ }^{9,10,13,14}$ which is similar to earlier work that recognized the importance of self-generated sheared flows in edge turbulence. ${ }^{15-17}$ An important aspect of the NTP is checking the validity of the variety of models used, along with benchmarking codes during the development phase. Of equal importantance are comparisons between gyrofluid and gyrokinetic approaches that can help improve our understanding of plasma turbulence since much of the existing insights are based on fluid type models and analogies with Navier-Stokes turbulence. Detailed comparisons of the linearized gyrofluid equations in the sheared-slab ${ }^{5}$ and toroidal ${ }^{6,9,18}$ systems show good agreement with linear gyrokinetic 
theory and there have been some preliminary nonlinear slab comparisons as well. ${ }^{13}$ Here, more complete and systematic nonlinear comparisons are made.

The ITG instability is used throughout this study. We will take the electrostatic limit and assume the electrons are adiabatic. The ITG mode has long been considered a plausible candidate for explaining the anomalous ion heat diffusivity in the core of tokamak plasmas, which is far above neoclassical predictions. ${ }^{19}$ We will begin by comparing a simple two dimensional (2D) shearless slab model where analytic comparisons can be made. We will then discuss fully turbulent three dimensional (3D) sheared slab comparisons, ${ }^{20}$ and finally 3D toroidal comparisons.

\section{A. Overview of Codes}

In this study five different codes will be compared: two gyrokinetic $\delta f$ particle codes (slab and toroidal), two gyrofluid codes (slab and toroidal), and a gyrokinetic Vlasov code (toroidal). A brief description of each of the codes follows, mentioning only the features used in the comparison study. More complete descriptions of the available options in the various codes can be found in the references.

The 3D gyrokinetic slab particle code ${ }^{21,22}$ is periodic in $y$ and $z$, and "bounded" in $x$ ( $\Phi=0$ at the boundaries). Both gyrokinetic codes use the partially linearized $\delta f$ scheme $\mathrm{s}^{23}$ in this comparison. The equilibrium density and temperature gradients and the magnetic shear are constant in time and vary in the $x$ direction. The 3D toroidal gyrokinetic code is similar to the slab code, but uses field-line-following coordinates ${ }^{18,24}$ to simulate a flux tube volume element. This code uses flux coordinate Hamiltonian guiding center equations 
of motion. ${ }^{25}$ Periodicity is assumed in all 3 directions in the field-line coordinates.

To facilitate detailed comparisons, the $3 \mathrm{D}$ slab gyrofluid code ${ }^{20}$ employs the same physical model as the 3D gyrokinetic slab code. The code solves three to eight fluid moments using pseudo-spectral methods. The 3D toroidal gyrofluid code $\mathrm{e}^{9,18}$ is similar to the slab code, but uses a field-line-following representation, includes toroidal drift resonances, and evolves four to six fluid moments. Toroidal and poloidal periodicity constraints are employed and the system is assumed periodic in the radial direction. Waltz and Kerbel [10] have made similar usage of field-line-following coordinates in a gyrofluid code.

The Vlasov code ${ }^{26,27}$ integrates the gyrokinetic equation ${ }^{1}$ in the 5 -dimensional phase space. The distribution function is represented on a $\mu, v_{\|}$grid in velocity space, and a $\theta$ grid (coordinate along the field line) with discrete $\theta_{0}$ 's and $k_{\theta}$ 's in ballooning space.

\section{B. Gyrofluid and Gyrokinetic Slab Equations}

For completeness, we write the electrostatic slab gyrokinetic equation for the perturbed ion distribution function $\delta f\left(\mathbf{x}, v_{\|}, t\right)$ integrated over $v_{\perp}$, taking $\left(k_{\perp} \rho_{i}\right)^{2} \rightarrow 0$ (the drift-kinetic limit), neglecting the parallel nonlinearity and assuming a Maxwellian background

$$
\partial_{t} \delta f+\mathbf{v}_{E} \cdot \nabla \delta f+v_{\|} \nabla_{\|} \delta f=-\left[1+\eta_{i} / 2\left(v_{\|}^{2}-1\right)\right] \partial_{y} \Phi f_{0}-v_{\|} \nabla_{\|} \Phi f_{0}
$$

using what we will term "gyrofluid units" with $\mathbf{x}_{\perp}=\mathbf{x}_{\perp} / \rho_{i}, x_{\|}=x_{\|} / L_{n}, v=v / v_{t i}$, $t=t v_{t i} / L_{n}, \Phi=e \Phi / T\left(L_{n} / \rho_{i}\right), \eta_{i}=L_{n} / L_{T}$, and $\delta f=\delta f L_{n} / \rho_{i}$. The quantity $\rho_{i}$ is the ion gyro-radius, $v_{t i}$ is the ion thermal velocity, $L_{n}$ is the density gradient scale length, $L_{T}$ is the temperature gradient scale length, and $\mathbf{v}_{E}=\hat{b} \times \nabla \Phi$. (See Ref. 28 for details.) The 
corresponding slab ion gyrofluid equations, ${ }^{5}$ in the $\left(k_{\perp} \rho_{i}\right)^{2}=0$ "Landau-fluid" limit are

$$
\begin{gathered}
\partial_{t} n+\mathbf{v}_{E} \cdot \nabla n+\nabla_{\|} u+\frac{\partial \Phi}{\partial y}=0 \\
\partial_{t} u+\mathbf{v}_{E} \cdot \nabla u+\nabla_{\|}[T+n+\Phi]=0, \\
\partial_{t} T+\mathbf{v}_{E} \cdot \nabla T+2 \nabla_{\|} u+\eta_{i} \frac{\partial \Phi}{\partial y}+\sqrt{\frac{8}{\pi}}\left|k_{\|}\right| T=0
\end{gathered}
$$

where $u(x, t)=\int v_{\|} \delta f d^{3} v /\left(n_{0} v_{t i}\right), T(x, t)=T_{\|}=\int\left(v_{\|}-u_{\|}\right)^{2} \delta f d^{3} v /\left(n_{0} v_{t i}^{2}\right)-n, n(x, t)=$ $\int \delta f d^{3} v / n_{0}$, and $n_{0}$ is the equilibrium density. We have taken $T_{i}=T_{e}$ throughout this comparison study since allowing $T_{i} \neq T_{e}$ is straightforward. See Refs. 5,28 for further details. For simplicity of comparison, we will set the $k_{y}=0, k_{z}=0$ component of $\Phi$ to zero in all of the 2-D and 3-D slab simulations (though not in the toroidal simulations, see Sec. 4), thus ignoring sheared poloidal flows which might be generated by the turbulence. Then the adiabatic electron response can be written as $n_{e}=\Phi$, and in the $k_{\perp} \rho_{s}=0$ limit the quasineutrality concition is just

$$
n=\Phi
$$

For the gyrokinetic system the density is obtained using $n=\int \delta f d^{3} v / n_{0}$. We will begin by comparing the gyrofluid system [Eqs. (2)-(5)] and the gyrokinetic system [Eqs. (1) and (5)] in the 2D slab model discussed below. More complicated comparisons then follow including finite gyroradius effects, higher moments and toroidal effects. All the $3 \mathrm{D}$ cases will include full finite gyroradius effects. Details on the more general equations can be found in Refs. $1-3,5,6,9$. 


\section{2D Shearless Slab Comparisons}

The first comparison will be with a simple "bounded 2D slab" model ${ }^{28}$ that greatly simplifies the comparison of the two simulations and allows for straightforward comparison with analytic theory. The system is taken as bounded in $x$ (i.e., $\Phi(x=0)=0, \Phi\left(x=L_{x}\right)=$ 0 ), periodic in $y$ and uniform in $z$. The magnetic field is uniform and has a small tilt in the $y$-direction, $\hat{b}=\hat{z}+\theta \hat{y}$, providing a finite (and constant) $k_{\|}=\theta k_{y}$. In the gyrofluid units, $k_{\|}=\left(\theta L_{n} / \rho_{i}\right) k_{y}$. To further simplify the nonlinear comparison, we neglect terms of order $\left(k_{\perp} \rho_{i}\right)^{2}$ as in Sec. I.B. Due to the boundary conditions in $x$ and $y, \Phi$ (as well as all the other field quantities) can be written as

$$
\Phi(x, y)=\sum_{\mathbf{k}} \Phi_{\mathbf{k}} \sin \left(k_{x} x\right) e^{i k_{y} y}
$$

where $\mathbf{k}=\left(k_{x}, k_{y}\right)=\left(\frac{\pi l}{L_{x}}, \frac{2 \pi m}{L_{y}}\right)$ for $l= \pm 1, \pm 2, \ldots$ and $m=0, \pm 1, \pm 2, \ldots$, with the following symmetry conditions satisfied: $\Phi_{-l, m}=-\Phi_{l, m}, \Phi_{l,-m}=\Phi_{l, m}^{*}$. To allow comparison with the analytic prediction of the saturation level we further simplify the problem by keeping only the following $(l, m)$ modes: $( \pm 1, \pm 1),( \pm 2,0)$. Starting with arbitrarily small initial conditions, the saturation of an isolated mode gives an indication of the initial saturation mechanism, neglecting the effect of self-generated poloidal shear flow. We emphasize that this is a simplified coherent nonlinear test problem, and the turbulent $3 \mathrm{D}$ simulations are qualititatively quite different, with many modes excited. Fully turbulent 3D cases will be discussed in Section III and IV.

The details of the time evolution are not shown here but can be found in Ref. 19, and are very similar to the results found in Ref. 28 , for a somewhat different set of parameters 
and assumptions.

\section{A. 2D Shearless Slab Simulation Comparison}

The physical parameters for this first comparison are $k_{x} \rho_{i}=k_{y} \rho_{i}=0.1, \eta_{i}=10$, $T_{e} / T_{i}=1$, and varying $k_{\|} L_{n}$. The quantity $\theta L_{n} / \rho_{i}$ was varied from 0.25 to 3.15 to check the validity of the results over a range of $k_{\|}$. The box size used for both simulations was $L_{x}=31.4 \rho_{i}$ and $L_{y}=62.8 \rho_{i}$ with a $32 \times 32$ grid. The gyrokinetic code used 16,384 particles. Both codes used a timestep of $\Delta t=0.5 L_{n} / v_{t i}$. The simulations were well converged using these parameters. Figure 1 shows the linear real frequency and growth rate vs. $\theta L_{n} / \rho_{i}$. There is excellent agreement between the two codes. The linear growth rate and real frequency is also shown from the kinetic plasma dispersion relation. ${ }^{20}$ Figure 2 shows the nonlinear saturation level for the two simulations, with reasonable agreement at small $k_{\|}$. The gyrofluid calculation, however, overestimates the saturation level at large $k_{\|}, e . g .2 .6$ times too large at $k_{\|}=\theta\left(L_{n} / \rho_{i}\right) k_{y}=0.24$. In both codes only the $\Phi( \pm 1, \pm 1), \Phi( \pm 2,0)$ modes were kept. However, all modes were kept in the other field quantities associated with $\delta f$. This was done because of difficulties with zeroing various Fourier modes of $\delta f$ in the gyrokinetic simulations. The comparisons with the two analytic results also shown in Fig. 2 will be discussed below.

For this comparison, we use the following definition of the ion thermal diffusivity $\chi_{i},{ }^{20}$ in gyrofluid units, $\chi_{i}=\langle Q\rangle / \eta_{i}=\left\langle\int \partial_{y} \Phi \frac{1}{2}\left(v_{\|}^{2}+v_{\perp}^{2}\right) \delta f d^{3} v\right\rangle / \eta_{i}$. Figure 3 shows the ion thermal diffusivity at the peak level in both simulations. In this model the flux grows exponentially in the linear phase, nonlinearly saturates, then drops to a small value. ${ }^{20,28}$ It is interesting 
that there is good agreement in $\chi_{i}$, even in the large $k_{\|}$regime when the saturation levels are quite different.

\section{B. Comparison with Gyrofluid Theory}

Next, we calculate the nonlinear saturation of the gyrofluid system. Following Lee and Tang, ${ }^{28}$ saturation occurs when the $( \pm 1, \pm 1)$ unstable mode couples to the $( \pm 2,0)$ temperature perturbation which results in a flattening of the equilibrium temperature profile. The present calculation is similar to Ref. 28 , but with the Landau term, ${ }^{4}$ finite $\eta_{i}$. From Eq. 6 , the $\mathbf{E} \times \mathbf{B}$ nonlinearity becomes

$$
\mathbf{v}_{E} \cdot \nabla u=\sum_{\mathbf{k}=\mathbf{k}^{\prime}+\mathbf{k}^{\prime \prime}}\left(k_{x}^{\prime} k_{y}^{\prime \prime}-k_{y}^{\prime} k_{x}^{\prime \prime}\right) \Phi_{\mathbf{k}^{\prime}} u_{\mathbf{k}^{\prime \prime}}
$$

Since we are taking the zero $k_{\perp} \rho_{i}$ limit, $n=\Phi$. It follows from Eq. (2) that $\Phi(2,0)=0$; hence there is no poloidal shear flow in this test problem. Using Eqs. (2)-(4) we obtain the following evolution equations for the $(1,1)$ mode (denoted by the subscript " 1 "):

$$
\begin{gathered}
\frac{\partial \Phi_{1}}{\partial t}+i k_{\|} u_{1}+i k_{y} \Phi_{1}=0 \\
\frac{\partial u_{1}}{\partial t}+i k_{\|}\left[T_{1}+2 \Phi_{1}\right]+i k_{\perp}^{2} \Phi_{1} u_{0}=0 \\
\frac{\partial T_{1}}{\partial t}+2 i k_{\|} u_{1}+\eta_{i} i k_{y} \Phi_{1}+\sqrt{\frac{8}{\pi}} k_{\|} T_{1}+i k_{\perp}^{2} \Phi_{1} T_{0}=0
\end{gathered}
$$

and the $(2,0)$ mode (denoted by the subscript " 0 "):

$$
\begin{aligned}
& \frac{\partial u_{0}}{\partial t}=2 k_{\perp}^{2} \operatorname{Im}\left(\Phi_{1}^{*} u_{1}\right), \\
& \frac{\partial T_{0}}{\partial t}=2 k_{\perp}^{2} \operatorname{Im}\left(\Phi_{1}^{*} T_{1}\right),
\end{aligned}
$$


where $k_{\perp}=2 k_{x} k_{y}$ (note the uncommon definition of $k_{\perp}$ ). In Eqs. (7)-(11) and the rest of this section $k_{x}, k_{y}, k_{\perp}$ and $k_{\|}$are evaluated for the $(1,1)$ mode. This set of equations, Eqs.. (9)-(11), has been solved numerically by an independent code providing a useful nonlinear benchmark for the much more complex slab gyrofluid simulation.

Using Eqs. (7)-(9) and dropping the nonlinear terms, the linear dispersion relation may be obtained:

$$
\left(\frac{\omega}{k_{\|}}+i \sqrt{\frac{8}{\pi}}\right)\left[2-\frac{\omega}{k_{\|}}\left(\frac{\omega}{k_{\|}}-\frac{k_{y}}{k_{\|}}\right)\right]+2 \frac{\omega}{k_{\|}}+\left(\eta_{i}-2\right) \frac{k_{y}}{k_{\|}}=0 .
$$

An analytic estimate of the saturation level and nonlinear frequency shift can be obtained by assuming $n_{1}, u_{1}$ and $T_{1}$ have $e^{-i \omega_{l} t+\gamma_{l} t}$ time dependence, where $\omega_{l}+i \gamma_{l}$ is the complex linear frequency. It then follows that $u_{0}$ and $T_{0}$ have $e^{2 \gamma_{l} t}$ time dependence.

Eqs. (7)-(11) then yield the following "quasi-linear dispersion relation" involving the real part of the linear frequency $\left(\omega_{l}\right)$ and the nonlinear $\left(\omega_{n l}\right)$ frequency

$$
\begin{gathered}
\left(\frac{\omega_{n l}}{k_{\|}}+i \sqrt{\frac{8}{\pi}}\right)\left[2-\frac{\omega_{n l}}{k_{\|}}\left(\frac{\omega_{n l}}{k_{\|}}-\frac{k_{y}}{k_{\|}}\right)+2 \frac{k_{\perp}^{4}}{k_{\|}^{2}}\left|\Phi_{1}\right|^{2}\right]+2 \frac{\omega_{n l}}{k_{\|}}+\left(\eta_{i}-2\right) \frac{k_{y}}{k_{\|}} \\
+\frac{k_{\perp}^{4}}{k_{\|}^{2}}\left|\Phi_{1}\right|^{2}\left(2 \frac{\omega_{l}}{k_{\|}}-\frac{k_{y}}{k_{\|}}\right)=0
\end{gathered}
$$

At the time of nonlinear saturation the imaginary part of the nonlinear frequency must vanish. Thus, the coefficient in square brackets on the right-hand side must be identically zero, giving the following expression for the saturation level:

$$
\left|\Phi_{1}\right|^{2}=\frac{k_{\|}^{2}}{k_{\perp}^{4}}\left[\frac{\omega_{n l}}{k_{\|}}\left(\frac{\omega_{n l}}{k_{\|}}-\frac{k_{y}}{k_{\|}}\right)-2\right] .
$$

The nonlinear frequency $\omega_{n l}$ can be obtained from substituting Eq. (14) back into Eq. (13) to obtain a simple quadratic equation. The result of solving for the saturation level is shown 
in Fig. 2 labeled "TMC Theory" (for Three Mode Coupling). There is a $20 \%$ difference, but the right scaling with $k_{\|}$is observed. Best agreement (within $5 \%$ ) is found if the gyrofluid code keeps only the $( \pm 1, \pm 1)$ and $( \pm 2,0)$ modes for the $n, u$ and $T$ moments (as stated above all the Fourier modes were kept for the moments to mimic the gyrokinetic simulation). To compare with conventional fluid theory, one simply drops the the $i \sqrt{8 / \pi}$ term in the dispersion relations, Eqs. (12) and (13). [In the strong instability regime of $\eta_{i} \gg 1$ and $\omega_{* T i} \gg k_{\|} v_{t i}$, this reduces to Eq. 40 in the earlier work of Ref. 28.] This term comes directly from the Landau damping model ${ }^{4}$ and is important for the good agreement with gyrokinetic linear theory. We also note that without the Landau-damping term in Eqs. (7)-(11), the mode amplitude time histories are quite different exhibiting strong regular nonlinear oscillations when numerically integrated.

\section{Comparison with Gyrokinetic Theory}

Following Lee et al. ${ }^{29}$ for drift waves, an analogus nonlinear calculation starting with Eqs. (1) and (5) can be carried out assuming a warm ion responce $\left(\omega_{l} / k_{\|} \ll v_{t i}\right)$ along with $\gamma_{l} / \omega_{l} \ll 1$ and $\eta_{i} \gg 1$. Although the most unstable ITG modes usually have the property of $\omega / k_{\|} \simeq v_{t i}$, this analysis can give us some important insight with regard to the scaling for nonlinear saturation. It involves the interaction of three Fourier modes, i.e., $\delta f(1, \pm 1)$ modes, which are complex conjugates of each other and are linearly unstable, and the nonlinearly-generated $\delta f(2,0)$ mode. For simplicity, we only consider $\phi(1, \pm 1)$ potential perturbations. It can then be shown that the saturation takes place when $\delta f(2,0)$ 
nonlinearly interferes with the growths of $\delta f(1, \pm 1)$ at an amplitude of

$$
|\phi|=\gamma_{l} / k_{\perp}^{2}
$$

For clarity, we write Eq. (15) in the dimensional form $\frac{e|\phi|}{T_{i}}=\frac{\gamma_{l}}{\omega_{* T}} \frac{1}{2 L_{T} k_{r}}$. This might appear to differ from the result found in Ref. 28 , that $|\phi| \simeq\left|\omega_{l}+i \gamma_{l}\right| / k_{\perp}^{2}$, but that result was only intended to apply to a regime far from marginal stability where $\eta_{i} \gg 2, \omega_{* T} \gg k_{\|} v_{t i}$, and $\omega_{l}+i \gamma_{l} \approx\left(\omega_{* T} k_{\|}^{2} v_{t i}^{2}\right)^{1 / 3}$. Thus, in the regime where it is applicable, $\left|\omega_{l}+i \gamma_{l}\right|$ differs from $\gamma_{l}$ only by $\sim 15 \%$. Since Eq. (15) is the same as for the drift wave case, ${ }^{29}$ the physical mechanism for the saturation can be understood through wave-particle trapping. ${ }^{30}$ The particle Hamiltonian in the wave frame takes the form ${ }^{31}$

$$
H=k_{x} k_{y} \phi+\left(k_{\|} v_{\|}-\omega\right) k_{x} x
$$

where $\phi$ is the electrostatic potential and $\omega$ is the wave frequency. The resonant particles with $k_{\|} v_{\|} \simeq \omega$, which are stationary relative to the wave, can become $\mathbf{E} \times \mathbf{B}$ trapped as the amplitude of $\phi$ grows in time. As a result, the resonant particles start to move along the contours of the constant $\phi$ and thereby shut off the growth. When this happens, the average $\mathbf{E} \times \mathbf{B}$ trapping frequency, $\omega_{t r} \simeq 2 k_{x} k_{y} \phi$, becomes comparable to the linear growth rate, $\gamma_{l}$, and the growth stops. We then recover the saturation criterion in Eq. (15). As shown in Fig. 2, there is good agreement especially in terms of scaling with $k_{\|}$. This scaling of the saturation amplitude was observed by Dorland $\mathrm{d}^{20}$ and has been confirmed numerically using a gyrokinetic Vlasov code, ${ }^{32}$ which solves the same set of equations in the Fourier-transform q-space for the velocities. ${ }^{33}$ While the particle simulations agree well with theory for all $k_{\|}$, the gyrofluid results overestimate the saturation level at large $k_{\|}$, 
where marginal stability is approached. This is reminiscent of the difficulties the gyrofluid eqs. have in the deeply-resonant weak-turbulence (near-marginal-stability) cases. ${ }^{34}$ One possible explanation for the discrepancy is that the effect of the $\mathbf{E} \times \mathbf{B}$ trapping is reduced in the gyrofluid model. Near marginal stability, the distribution function develops a sharp resonant structure in velocity space as expected theoretically and confirmed using Vlasov simulations. If only a few fluid moments are kept, the gyrofluid equations are only able to model this coherent resonant behavior in a very rough way ( $\Phi$ does drop to zero as marginal stability is approached, but not in the same way as the particle simulation). A more accurate result could be obtained if many fluid moments were kept [equivalent to keeping many terms in a Hermite polynomial expansion of $f(v)^{33}$. However, this sharp resonant structure is quite sensitive to collisions which smooth out fine-scale structure in velocity space via the $\nu v_{t}^{2} \partial^{2} f / \partial v^{2}$ term, in a similar fashion as for drift waves. ${ }^{32}$ Specifically, collisions can diminish and eventually nullify the trapping and as a result, increase the saturation level. The implications for the more realistic turbulent $3 \mathrm{D}$ system is not clear. It might be expected that the effects of coherent trapping would be reduced for a many mode system due to stochastic $\mathbf{E} \times \mathbf{B}$ motion and resonance broadening, along with finite collisionality. This may lead to better agreement between the two approaches in the more realistic turbulent systems.

\section{Turbulent 3D Slab Comparisons}

In this section we will compare the codes using a turbulent $3 \mathrm{D}$ sheared slab test problem. The system is bounded in $x$ and periodic in $y$ and $z$. The magnetic field direction $\hat{b}=\hat{z}+\frac{x}{L_{s}} \hat{y}$ 
has constant shear, with $x=0$ being in the center of the the box. The bounded box avoids complications of handling the shift in the direction in $\hat{b}$ at the ends of the box $\left(x= \pm L_{x} / 2\right)$. The radial boundary conditions were chosen to simplify the code comparison, but not on physical grounds. The system is large enough, however, making the transport time scale long enough to allow for a reasonable quasi-steady state.

The local physical parameters are shown in Table 1 and were taken from a TFTR Lmode shot (termed the the "NTP test case parameters"). ${ }^{35}$ The $k_{y}=0, k_{z}=0$ modes were suppressed for this comparison. The gyrokinetic results shown used a system size of $L_{x}=51.2 \rho_{i}, L_{y}=51.2 \rho_{i}, L_{z}=25.6 L_{n}$, a $128 \times 128 \times 64$ grid, and 8 particles per grid cell. Modes up to $k_{x} \rho_{i}=1.5, k_{y} \rho_{i}=2, k_{z} L_{n}=2$ were well resolved, and the timestep was $\Delta t v_{t i} / L_{n}=0.125$. These runs are extremely well resolved with similar results being obtained using 4 particles per grid cell on a $128 \times 64 \times 64$ grid resolving up to $k_{x} \rho_{i}=1.5$, $k_{y} \rho_{i}=1$, and $k_{z} L_{n}=2$. The gyrofluid simulation used the same box size (so spacing between rational surfaces was the same in both simulations) with a $128 \times 32 \times 32$ grid resolving up to $k_{x} \rho_{i}=1.5, k_{y} \rho_{i}=1$, and $k_{z} L_{n}=2$, and an average timestep of 0.04 .

Figure 4 shows the $k_{y}$ wavelength spectrum from the two simulations. The $k_{y}$ spectrum is summed over all $k_{x}$ and $k_{z}$ and time averaged. They both peak in the same range $k_{y} \rho_{i}=0.2$ and show little activity for $k_{y} \rho_{i} \gtrsim 0.8$. However, there is a $30-40 \%$ difference in the overall saturation level of $\Phi$. Figure 5 shows the ion heat diffusivity as a function of magnetic shear. The ion diffusivity is time averaged and measured after a quasi-steady state is reached. Details of the time histories can be found in Ref. 20. The overall level, as well as the scaling with magnetic shear, seem quite good. The results shown in Fig. 5 
do not include self-generated shear flow which is an important effect for weaker magnetic shear cases when using a simple adiabatic electron model. ${ }^{20}$

\section{A. Field Line Following Simulations in the Slab Limit}

Next, we compare the field line following coordinate simulations in the slab limit. The coordinate system used is given in Ref. $24\left(x^{\prime}=x, y^{\prime}=y-x z / L_{s}, z^{\prime}=z\right)$. The system is taken to be periodic in the primed coordinates. The results are tabulated using the NTP test-case parameters in Table 2, where the rms $\Phi$ is defined by $(\operatorname{rms} \Phi)^{2}=\left\langle\Phi^{2}\right\rangle=\sum_{k}\left|\Phi_{k}\right|^{2}$. The linear agreement is very good between all the codes with the peak growth rate found at $k_{y} \rho_{i}=0.49$. The field-line-following coordinate codes are designated by "FLF." The gyrokinetic code used a box size in primed coordinates of $L_{x}^{\prime}=51.2 \rho_{i}, L_{y}^{\prime}=51.2 \rho_{i}$, and $L_{z}^{\prime}=160 L_{n}$, with a $64 \times 32 \times 64$ grid, 4 particles per grid cell, $\Delta t v_{t i} / L_{n}=0.025$, and a Fourier filter of $\exp \left(-k_{x}^{\prime 4} \rho_{i}^{4}-k_{y}^{\prime 4} \rho_{i}^{4}\right)$. When only $k_{\|}=0, k_{y}=0$ modes are set to zero, the particle code did not achieve a saturated steady state. This is noted in the Table 2 as "n.s." for no saturation. The code reaches a steady-state in a similar fashion as the gyrokinetic slab code when all the zero Fourier modes (for any $k_{x}^{\prime}, k_{x}^{\prime}, k_{z}^{\prime}$ ) are set to zero and this result is shown in the parenthesis. The reason for the lack of a steady-state when these other zero modes are kept needs to be investigated further. The gyrofluid code also used a $64 \times 32 \times 64$ grid, accurately resolving all modes up to $k_{x}^{\prime} \rho_{i}=k_{y}^{\prime} \rho_{i}=1$, and a timestep of $\Delta t v_{t i} / L_{n}=0.05$. When run in the slab limit, the toroidal gyrofluid code is in reasonable agreement with the slab gyrokinetic and gyrofluid codes (see Table 2). For this case, the $k_{\|}=0, k_{y}=0$ modes were set to zero to compare with the slab code results, although radial periodic boundary 
conditions were used. This code was also run with all the zero Fourier modes set to zero to compare to the toroidal gyrokinetic code in the slab limit with these results shown in parentheses. We caution the reader that differences between the field-line-following and slab codes should be expected due to the difference in the radial boundary conditions (periodic vs. bounded with the profile flattening).

\section{3D Toroidal Comparisons}

Finally we make linear and nonlinear comparisons including the toroidal drifts and magnetic mirroring. The gyrofluid, gyrokinetic $\delta f$ particle and gyrokinetic Vlasov codes simulate a small fraction of the tokamak plasma volume with the simulation domain being a flux tube. The field line following coordinates used in these simulations are $\mathrm{e}^{24,18}$

$$
x^{\prime}=r-r_{0}, \quad y^{\prime}=\frac{r_{0}}{q_{0}}(q(r) \theta-\phi), \quad z^{\prime}=R q(r) \theta
$$

where $r$ is the minor radius, $\theta$ is the poloidal angle, $\phi$ is the toroidal aingle, $q$ is the safety factor, $q_{0}=q\left(r_{0}\right)$, and $R$ is the major radius. The width of the box in the radial direction is assumed small compared to $r_{0}, d q / d r$ is assumed constant, and a zero $\beta$ unshifted circular magnetic equilibrium and small $r_{0} / R$ are also assumed. The domain is rectilinear in the field-line-following coordinates. Figure 6 shows a linear comparison of the toroidal simulations, using the NTP parameters shown in Table 1. There is reasonable agreement between the three different calculations. Possible improvements in the agreement between the Vlasov and particle $\delta f$ codes may be found by increased resolution in $z^{\prime}$ and/or implementation of proper toroidal periodicity in the particle code. These discrepancies will be 
investigated in the future. Dropping the $\mu \nabla B$ mirror force in the calculation changes these linear results very little (less than $10 \%$ ) for these L-mode type parameters.

For the nonlinear toroidal comparisons, the $m=0, n=0$ mode was kept using $\delta n_{e}(m=0, n=0)=0$, i.e. using the electron response $n_{e}=\Phi-\langle\Phi\rangle .^{5,9,13,18}$ FLR effects in the Poisson Eq., particularly the ion polarization density, ${ }^{2}$ are very important for determining this component of $\Phi$. Including this mode and the associated poloidal shear flows is important for achieving a saturated steady-state in the toroidal gyrofluid ${ }^{18}$ and gyrokinetic field-line-following codes with adiabatic electrons. The parameters are the same as in the slab comparison (Sec. III.A) except now with $L_{z}^{\prime}=2 \pi R$. The gyrokinetic code uses a timestep of $\Delta t v_{t i} / L_{n}=0.025$ and sets the the $m=0, n \neq 0$ modes to zero. The gyrofluid code also uses a timestep of $\Delta t v_{t i} / L_{n}=0.01$. The rins $\Phi$ and ion diffusivity are shown in Table 3. Similar results (within a factor of two) have been reported by Waltz using a simpler gyrofluid model. ${ }^{36}$

Both simulations exhibit a dominant $m=0, n=0$ mode. However, this mode is more pronounced in the gyrokinetic simulation, which is, in part, responsible for the higher gyrokinetic saturation level. In the future, the mechanisms for reducing the $m=0, n=0$ mode, i.e. drift-orbit averaging, will be compared between the two models. Also, the effects of improved resolution, proper toroidal periodicity, and including the $m=0, n \neq 0$ modes in the gyrokinetic simulation need to be explored further. This preliminary comparison shows agreement within a factor of two between the gyrofluid and gyrokinetic toroidal results, which is encouraging. However, we caution that this is only one test case (one set of physical parameters) and is not yet conclusive. 


\section{Summary}

A lot of attention to the details is necessary to get the agreement between gyrofluid and gyrokinetic simulations that was reported here. One must be careful to use the same boundary conditions (which affect whether the turbulence is maintained or eventually decays due to flattening of background gradients), similar resolution, rational surface spacing (which can affect local flattening around particular rational surfaces if they are too widely spaced), and the same treatment of the $k_{\theta}=k_{\|}=0$ component of $\Phi$ which corresponds to turbulence-generated sheared flows. One must also make sure that the two codes are consistent with each other, e.g. are using the same factors of $2, \pi$, etc. in all definitions.

The results reported here (with the slab results explained in more detail in Ref. 20), provide the first nonlinear tests of the gyrofluid models in fully turbulent 3-D systems. The results are generally encouraging, and indicate that models of phase-mixing, Landaudamping, and FLR effects in the gyrofluid equations ${ }^{5}$ may continue to work fairly well even in nonlinear turbulent systems. The slab codes have found excellent numerical agreement in the scaling of the turbulent heat diffusivity $\chi_{i}$ with magnetic shear (Fig. 5). However, there are some puzzling areas where more work can still be done. There are $\sim 40 \%$ differences in

the rms. $\Phi$ between the gyrofluid and gyrokinetic simulations, though similar qualitative scalings have been demonstrated. The gyrofluid saturation level was found to be higher than the gyrokinetic result in the coherent $2 \mathrm{D}$ slab test problem, where as, it was lower in the turbulent 3D slab case. In toroidal geometry, the field-line-following gyrofluid and gyrokinetic codes are within $\sim 50 \%$ of each other in the turbulent $\chi_{i}$ and $\operatorname{rms} \Phi$, though there is more work which can be done to investigate sensitivity to small differences in 
filtering and boundary conditions.

Perhaps most importantly, both approaches confirm important physics results: 1) the observation $^{8-10}$ that the turbulent spectrum peaks at significantly longer wavelengths than the linearly most-unstable modes, along with producing spectra with a shape similar to TFTR measurements ${ }^{8,11,12}$; and 2) the importance of turbulent-generated sheared flows even in core ITG simulations. ${ }^{9,10,13,14}$ This is similar to earlier work that recognized the importance of self-generated sheared flows in edge turbulence. ${ }^{15-17}$ More work needs to done on the effects of these flows, in particular to insure that all of the important damping and driving mechanisms for the flows are being properly modeled. Future work with larger simulation volumes needs to be done to find what controls the scaling of the long-wavelength features in the spectra, to explore puzzling experimental results such as Bohm-like scaling ${ }^{37}$ and the minor radius dependence of $\chi_{i},{ }^{38}$ and to extend the codes to include trappedelectrons, electromagnetic perturbations, and other mechanisms needed for a realistic model of tokamak turbulence.

\section{Acknowledgments}

This work benefited from useful collaborations with J.A. Byers, B.I. Cohen and A.M. Dimits and R.E. Waltz. We thank L. Chen, H.E. Mynick, J.A. Krommes, C.Z. Cheng, S.C. Cowley, T.S. Hahm and W.M. Tang for helpful discussions. Much of this work was carried out on computers at the National Energy Research Supercomputer Center at Lawrence Livermore National Laboratory. Work supported by the U.S. Department of Energy Contract No. DE-AC02-76-CHO-3073. 


\section{References}

${ }^{1}$ E.A. Frieman and L. Chen, Phys. Fluids 25502 (1982).

${ }^{2}$ W.W. Lee, Phys. Fluids 26556 (1983).

${ }^{3}$ T.S. Hahm, Phys. Fluids 312670 (1988).

${ }^{4}$ G. W. Hammett and F.W. Perkins, Phys., Rev. Lett. 643019 (1990).

${ }^{5}$ W. Dorland and G. W. Hammett, Phys. Fluids B 5812 (1993).

${ }^{6}$ R.E. Waltz, R.R. Dominguez and G.W. Hammett, Phys. Fluids B 43952 (1992).

${ }^{7}$ See National Technical Information Service Document No. UCRL-TB-111084 (D.C. Barnes, B.A. Carreras, B.I. Cohen, J.M. Dawson, G.W. Hammett, S.C. Jardin, G.D. Kerbel, P.C. Liewer, T. Tajima, and R.E. Waltz, "The Numerical Tokamak Project", in "Computing at the Leading Edge: Research in the Energy Sciences", Lawrence Livermore National Laboratory, A.A. Mirin, Ed, 1993). Copies may be obtained from the National Technical Information Service, Springfield, Virginia 22161.

${ }^{8}$ S.E. Parker, W.W. Lee and R.A. Santoro, Phys., Rev. Lett. 712042 (1993).

${ }^{9}$ G. W. Hammett, M. A. Beer, W. Dorland, S. C. Cowley, and S. A. Smith, Plasma Phys. Control. Fusion 35, 973 (1993).

${ }^{10}$ R.E. Waltz and G.D. Kerbel, Proc. U.S.-Japan Workshop on Ion Temperature Gradient Driven Turbulent Transport, Austin, TX, (New York, American Institute of Physics) to be published. 
${ }^{11}$ R. Fonck, N. Bretz, G. Cosby, R. Durst, E. Mazzucato, R. Nazikian, S. Paul, S. Scott, W.Tang, and M. Zarnstorff, Phys., Rev. Lett. 703736 (1993).

${ }^{12}$ E. Mazzucato and R. Nazikian, Phys., Rev. Lett. 711840 (1993).

${ }^{13}$ W. Dorland, G.W. Hammett, T.S. Hahm, and M.A. Beer Proc. U.S.-Japan Workshop on Ion Temperature Gradient Driven Turbulent Transport, Austin, TX, (New York, American Institute of Physics) to be published.

${ }^{14}$ B.I. Cohen, T.J. Williams, A.M. Dimits, and J.A. Byers, Phys. Fluids B 52967 (1993).

${ }^{15}$ A. Hasegawa, and M. Wakatani, Phys. Rev. Lett., 591581 (1987).

${ }^{16}$ B.A. Carreras, V.E. Lynch, and L. Garcia, Phys. Fluids B 31438 (1991).

${ }^{17}$ P.H. Diamond, and Y.B. Kim, Phys. Fluids B 31626 (1991).

${ }^{18}$ M.A. Beer, G.W. Hammett, W. Dorland, and S.C. Cowley, Bull. Am. Phys. Soc. 37, 1478 (1992).

${ }^{19}$ R.J. Fonck, R. Howell, K. Jaehnig, L. Roquemore, G. Schilling, S. Scott, M.C. Zarnstorff, C. Bush, R. Goldston, H. Hsuan, D. Johnson, A. Ramsey, J. Schivell, and H. Towner, Phys. Rev. Lett., 63520 (1989).

${ }^{20}$ W. Dorland, Ph. D. Thesis, Princeton University, (1993).

${ }^{21}$ R.A. Santoro and W.W. Lee, Bull. Am. Phys. Soc., Nov. (1993).

${ }^{22}$ W.W. Lee , J. Comput. Phys. 72, 243 (1987). 
${ }^{23}$ A.M. Dimits and W.W. Lee, J. Comput. Phys. 107, 309 (1993).

${ }^{24}$ S.C. Cowley, R.M. Kulsrud and R. Sudan, Phys. Fluids B 132767 (1991).

${ }^{25}$ R.B. White amd M.S. Chance, Phys. Fluids 272455 (1984).

${ }^{26}$ Q.P. Liu and C.Z. Cheng, Bull. Am. Phys. Soc., Nov, (1993).

${ }^{27}$ C.Z. Cheng and G. Knorr, J. Comput. Phys. 22, 330 (1976).

${ }^{28}$ W.W. Lee and W.M. Tang, Phys. Fluids, $31612(1988)$.

${ }^{29}$ W.W. Lee, J.A. Krommes, C.R. Oberman, and R.A. Smith, Phys. Fluids 272652 (1984).

${ }^{30}$ W.M. Manheimer, Phys. Fluids 14579 (1971).

${ }^{31}$ R.A. Smith, J.A. Krommes, and W.W. Lee, Phys. Fluids 281069 (1985).

${ }^{32}$ J.F. Frederici, W.W. Lee, and W.M. Tang, Phys. Fluids 302652 (1987).

${ }^{33}$ T.P. Armstrong, R.C. Harding, G. Knorr, and D. Montgomery in Methods of Computational Physics edited by B. Alder,S. Fernbach and M. Rotenberg, Academic Press, New York, 929 (1970).

${ }^{34}$ N. Mattor, Phys. Fluids B 43952 (1992).

${ }^{35}$ G.W. Hammett, and M. Zarnstorff private communication (1992); B.I. Cohen and R.E. Waltz, private communication (1993); S.D. Scott, V. Arunasalam, C.W. Barnes, M.G. Bell, H. Biglari, M. Bitter , N.L. Bretz, R. Budny, C.E. Bush, A. Cavallo, L. Chen, S.A. Cohen, P. Colestock, T.K. Chu, P.H. Diamond, S.L. Davis, D.L. Dimock, 
H.F. Dylla, P.C. Efthimion, A.B. Ehrhardt, E. Fredrickson, R.J. Fonck, H.P. Furth, G. Gammel, R.J. Goldston, G. Greene, B. Grek, L.R. Grisham, R. Boivin, D.J. Hoffman, R. Little, G.W. Hammett, R.J. Hawryluk , H.W. Hendel, K.W. Hill, E. Hinnov, J. Hosea, B. Howell , R.A. Hulse, H. Hsuan, K.P. Jaehnig, A.C. Janos, D.L. Jassby, F.C. Jobes, D.W. Johnson, L.C. Johnson, R. Kaita , S.J. Kilpatrick, P.H. LaMarche, B. LeBlanc, D.M. Manos, D. K. Mansfield, J.A. Murphy, D.C. McCune, K.M. McGuire, D.M. Meade, S.S. Medley, D.R. Mikkelsen, R. Motley, D. Mueller, Y. Nagayama, R. Nazakian, E.B. Nieschmidt, D.K. Owens, H. Park, C. Kieras-Phillips, N. Pomphrey, A.T. Ramsey, M.H. Redi , G. Rewoldt, A.L. Roquemore, G. Schilling, J. Schivell, G. L. Schmidt, S. Sesnic, J. Sinnis, J.E. Stevens, W. Stodiek, B. Stratton, J. Strachan, E.J. Synakowski, W. Tang, G. Taylor, M.E. Thompson, J. Terry, J.R. Timberlake H. H. Towner, M. Ulrickson, S. von Goeler, R.M. Wiel, M.D. Williams, K.L. Wong, S. Yoshikawa, K.M. Young, M.C. Zarnstorff, S.J. Zweben, Phys. Fluids B 21300 (1990).

${ }^{36}$ R.E. Waltz, G.D. Kerbel, and J. Milovich, "Toroidal gyro Landau fluid model turbulence simulations in a nonlinear ballooning mode representation with radial modes" General Atomics Report GA-A21565 to be submitted to Physics of Plasmas.

${ }^{37}$ F.W. Perkins, C.W. Barnes, D.W. Johnson, S.D. Scott, M.C. Zarnstorf, M.G. Bell, R.E. Bell. C.E. Bush, B. Grek, K.W. Hill, D.K. Mansfield, H. Park, A.T. Ramsey, J. Schivell, B.C. Stratton, and E. Synakowski, Phys. Fluids B 5477 (1993).

${ }^{38}$ P.C. Efthimion, D.K. Mansfield, B.C. Stratton, E. Synakowski, A. Bhattacharjee, H. Biglari, P.H. Diamond, R.J. Goldston, C.C. Hegna, D. McCune, G. Rewoldt, S. Scott, 
W.M. Tang, G. Taylor, R.E. Waltz, R.M. Wieland and M.C. Zarnstorff Phys. Rev. Lett. 66421 (1991).

\section{Figures}

Figure 1: Linear growth rate and real frequency vs. $\theta L_{n} / \rho_{i}=k_{\|} L_{n} /\left(k_{y} \rho_{i}\right)$ for the 2D shearless slab comparison. Results from the gyrofluid simulation, gyrokinetic simulation and the kinetic and conventional fluid linear dispersion relations.

Figure 2: Saturation level of the electrostatic potential vs. $\theta L_{n} / \rho_{i}=k_{\|} L_{n} /\left(k_{y} \rho_{i}\right)$ for $2 \mathrm{D}$ shearless slab comparison. Gyrofluid and gyrokinetic simulation results and theoretical predictions.

Figure 3: Ion thermal diffusivity measured at the peak level for $2 \mathrm{D}$ shearless slab comparison.

Figure 4: Comparison of $k_{y}$ wavelength spectrum for the turbulent 3D sheared test case.

Figure 5: Comparison of ion thermal diffusivity for the turbulent 3D sheared slab test case.

Figure 6: Comparison of toroidal linear growth rate and real frequency vs. $k_{\theta} \rho_{i}$. Results from gyrofluid, Vlasov and $\delta f$ particle codes. 


\begin{tabular}{|l|cccc|}
\hline Slab Parameters: & $\eta_{i}=L_{T} / L_{n}=4$ & $L_{s} / L_{n}=4$ & $T_{e} / T_{i}=1$ & \\
\hline Toroidal Parameters: & $r / R=0.205$ & $L_{n} / R=0.4$ & $q=2.4$ & $\hat{s}=r q^{\prime} / q=1.5$ \\
\hline
\end{tabular}

Table 1: Local physical NTP test case parameters.

\begin{tabular}{|l|c|c|c|}
\hline Codes & Linear $\omega$ & $\mathrm{rms} e \Phi / T\left(L_{n} / \rho_{i}\right)$ & $\chi_{i} L_{n} /\left(\rho_{i}^{2} v_{t i}\right)$ \\
\hline \hline GK Slab & $-0.20+0.060 i$ & 1.2 & 0.12 \\
\hline GF Slab & $-0.20+0.054 i$ & 0.83 & 0.13 \\
\hline \hline GK FLF & $-0.20+0.060 i$ & n.s. $(1.0)$ & n.s. $(0.13)$ \\
\hline GF FLF & $-0.20+0.054 i$ & $1.3(0.55)$ & $0.13(0.063)$ \\
\hline "Vlasov" FLF & $-0.19+0.070 i$ & - & - \\
\hline
\end{tabular}

Table 2: Comparison of the codes in the 3D sheared slab limit using the NTP parameters. The linear $\omega$ was taken at the most unstable mode which was $k_{\theta} \rho_{i}=0.49$ for all the codes. More details are given in the text. 


\begin{tabular}{|l|c|c|c|}
\hline Codes & Linear $\omega$ & $\operatorname{rms~} e \Phi / T\left(L_{n} / \rho_{i}\right)$ & $\chi_{i} L_{n} /\left(\rho_{i}^{2} v_{v t i}\right)$ \\
\hline \hline GK FLF & $-0.75+0.19 i$ & 16. & 2.9 \\
\hline GF FLF & $-0.67+0.18 i$ & 10. & 4.0 \\
\hline "Vlasov" FLF & $-0.61+0.18 i$ & - & - \\
\hline
\end{tabular}

Table 3: Toroidal code comparison using the NTP parameters. Linear $\omega$ measured at $k_{\theta} \rho_{i}=0.49$. 


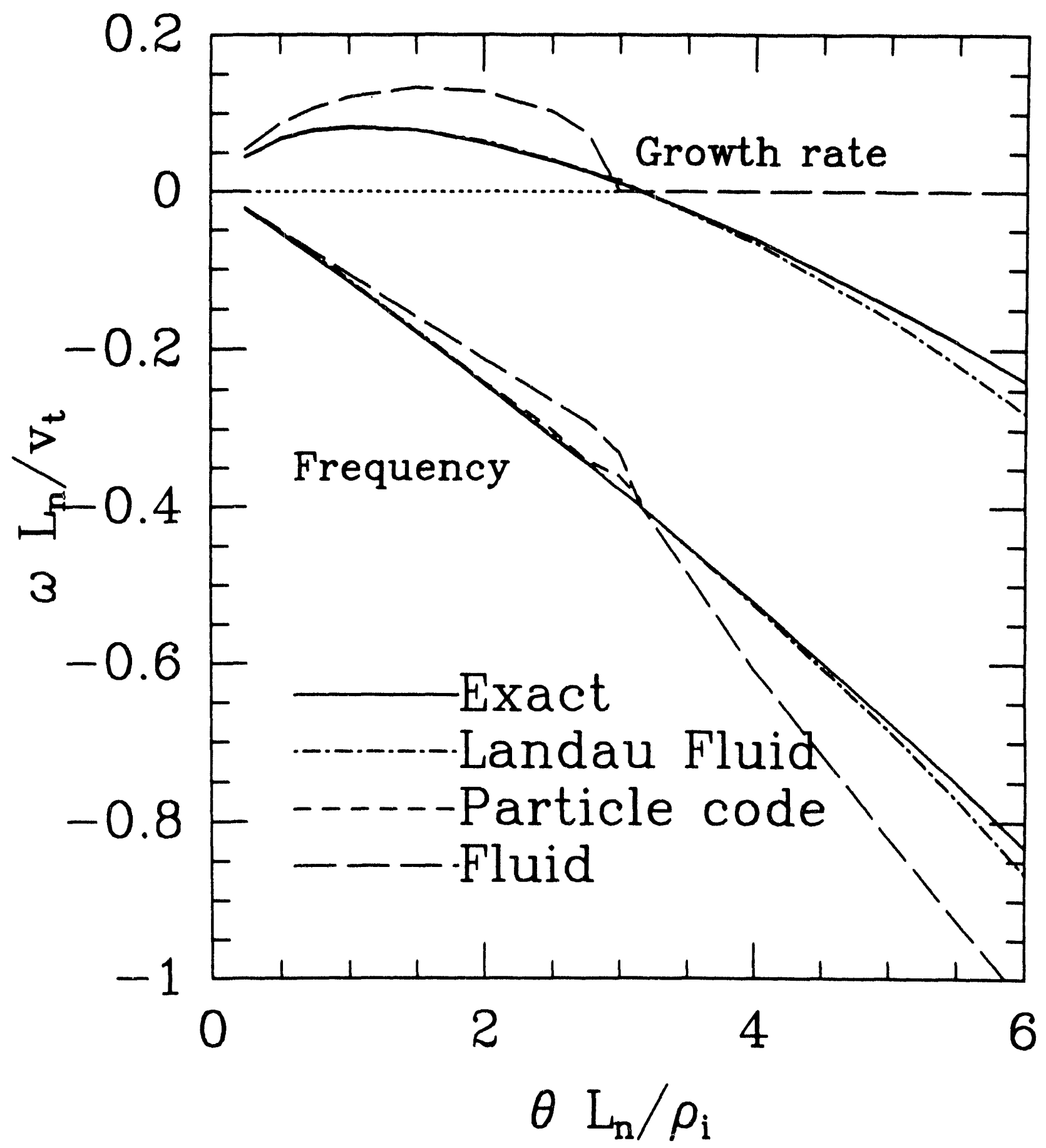

Figure 1 


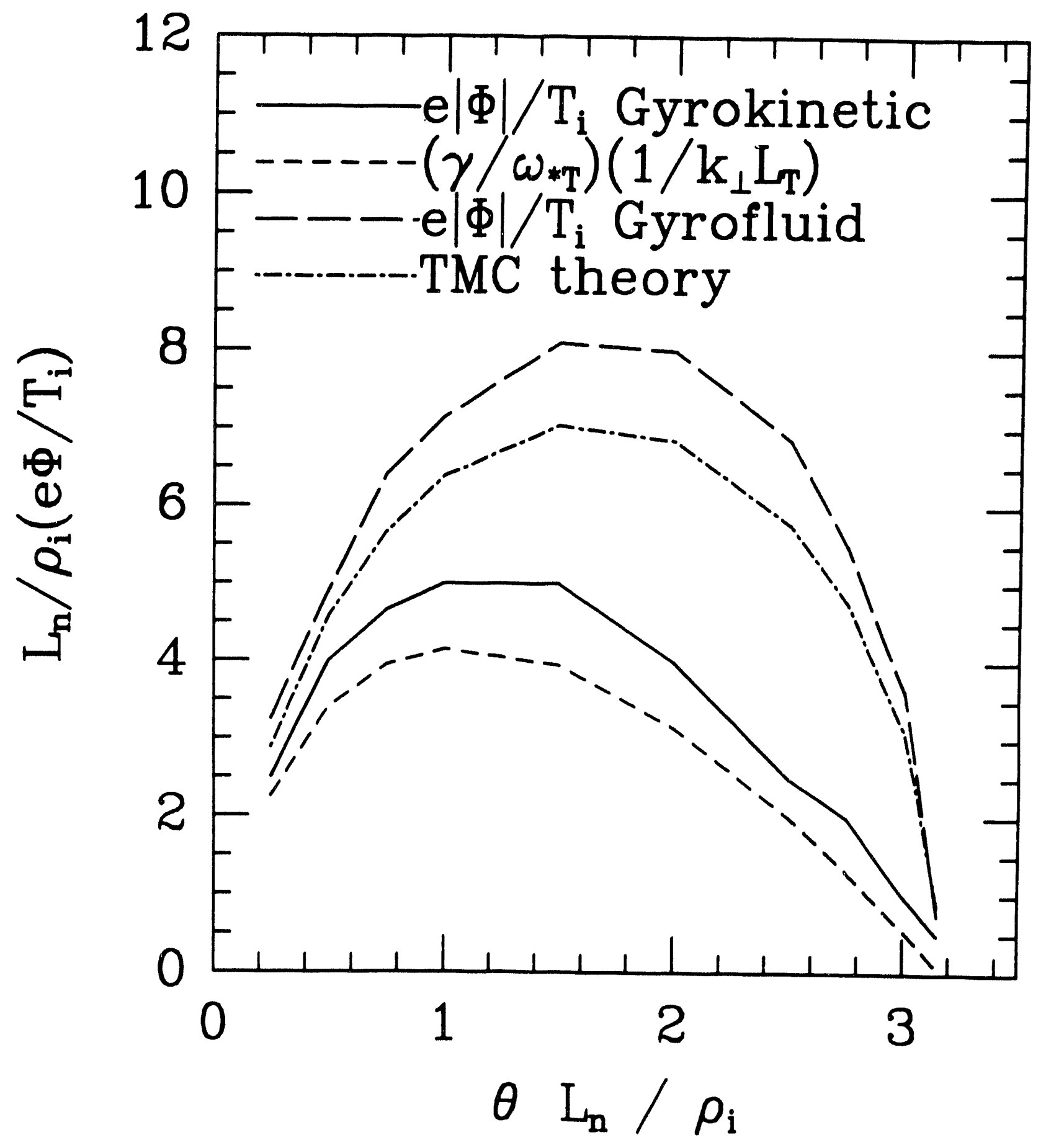

Figure 2 


\section{Thermal Diffusivity}

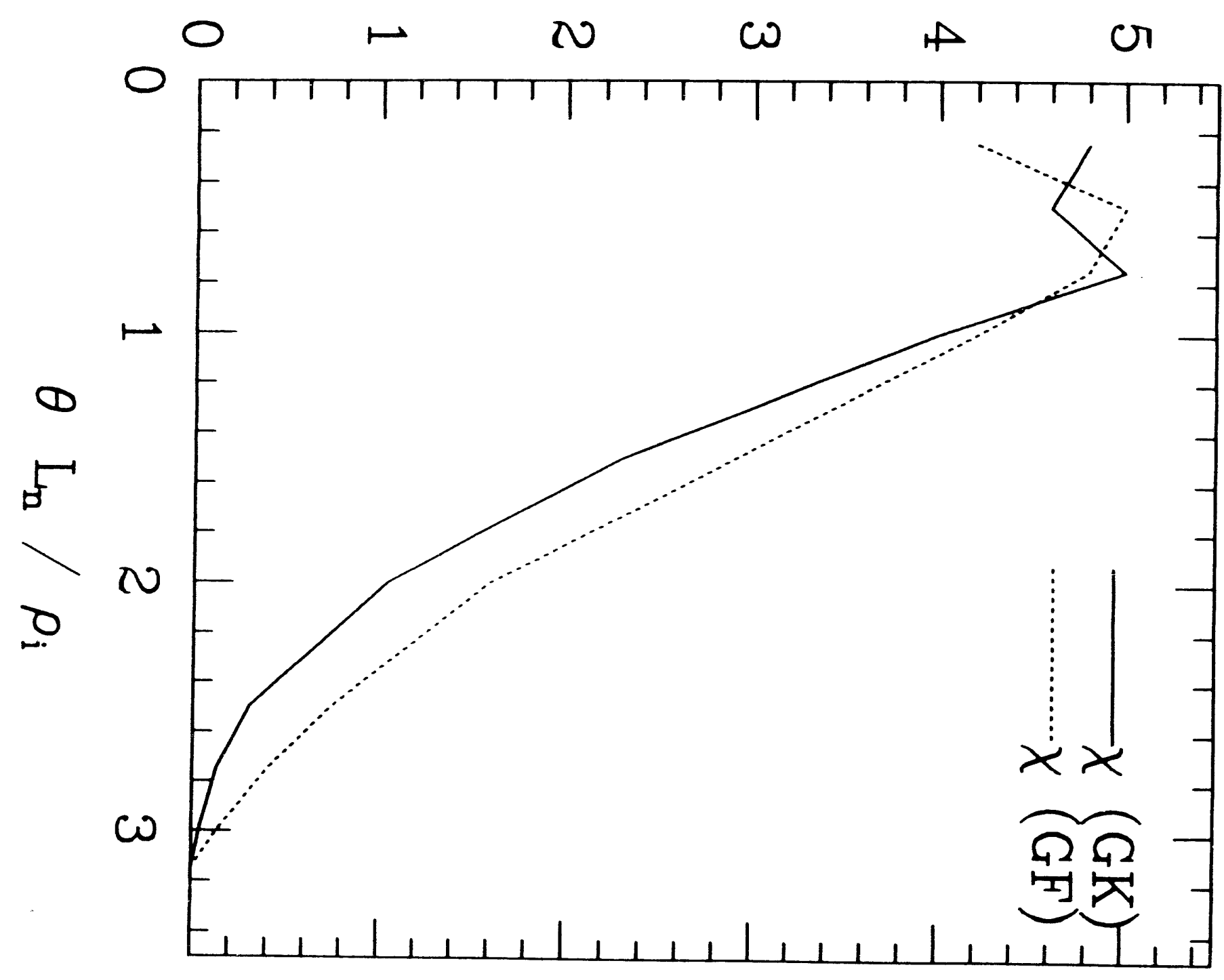




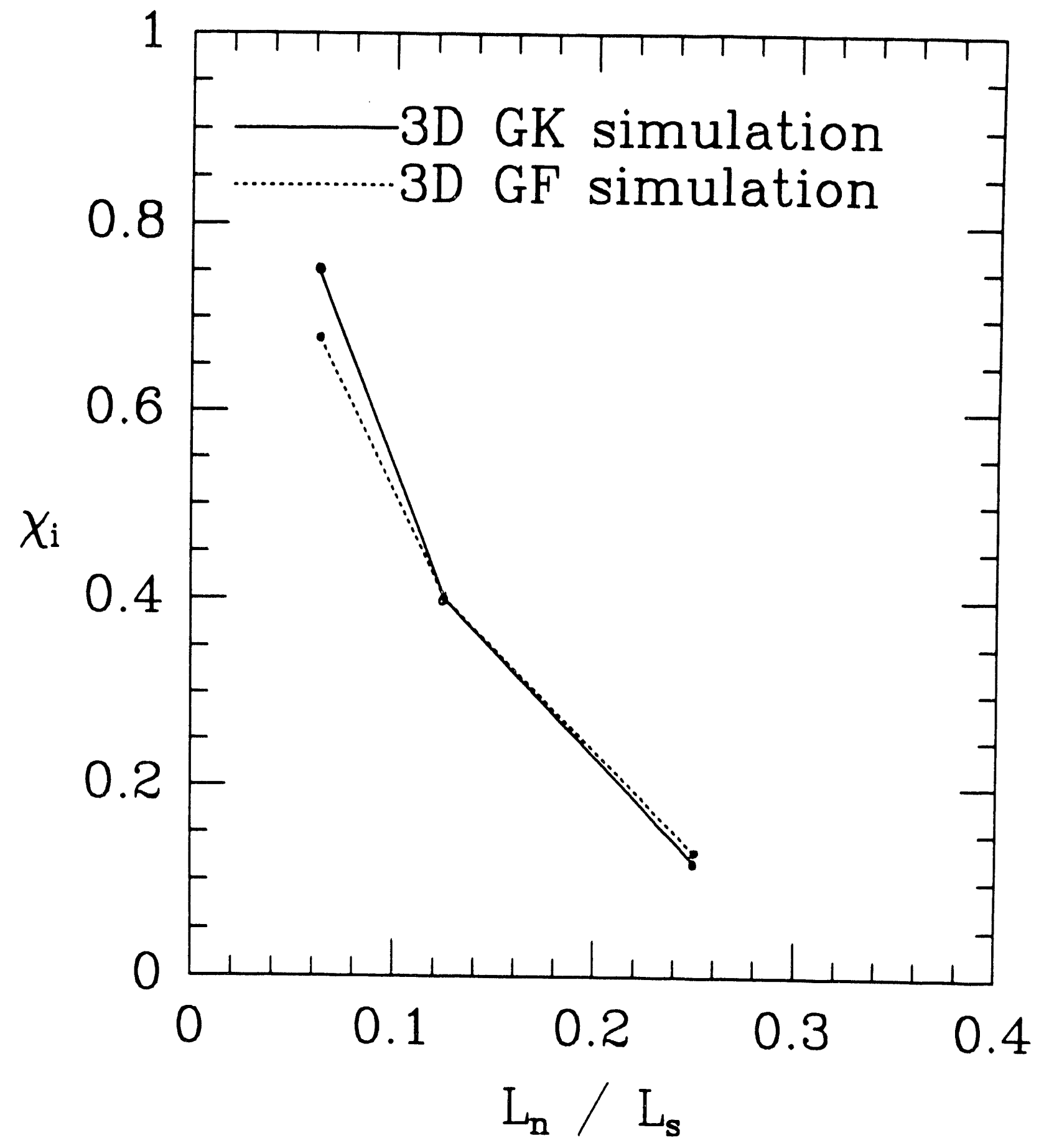



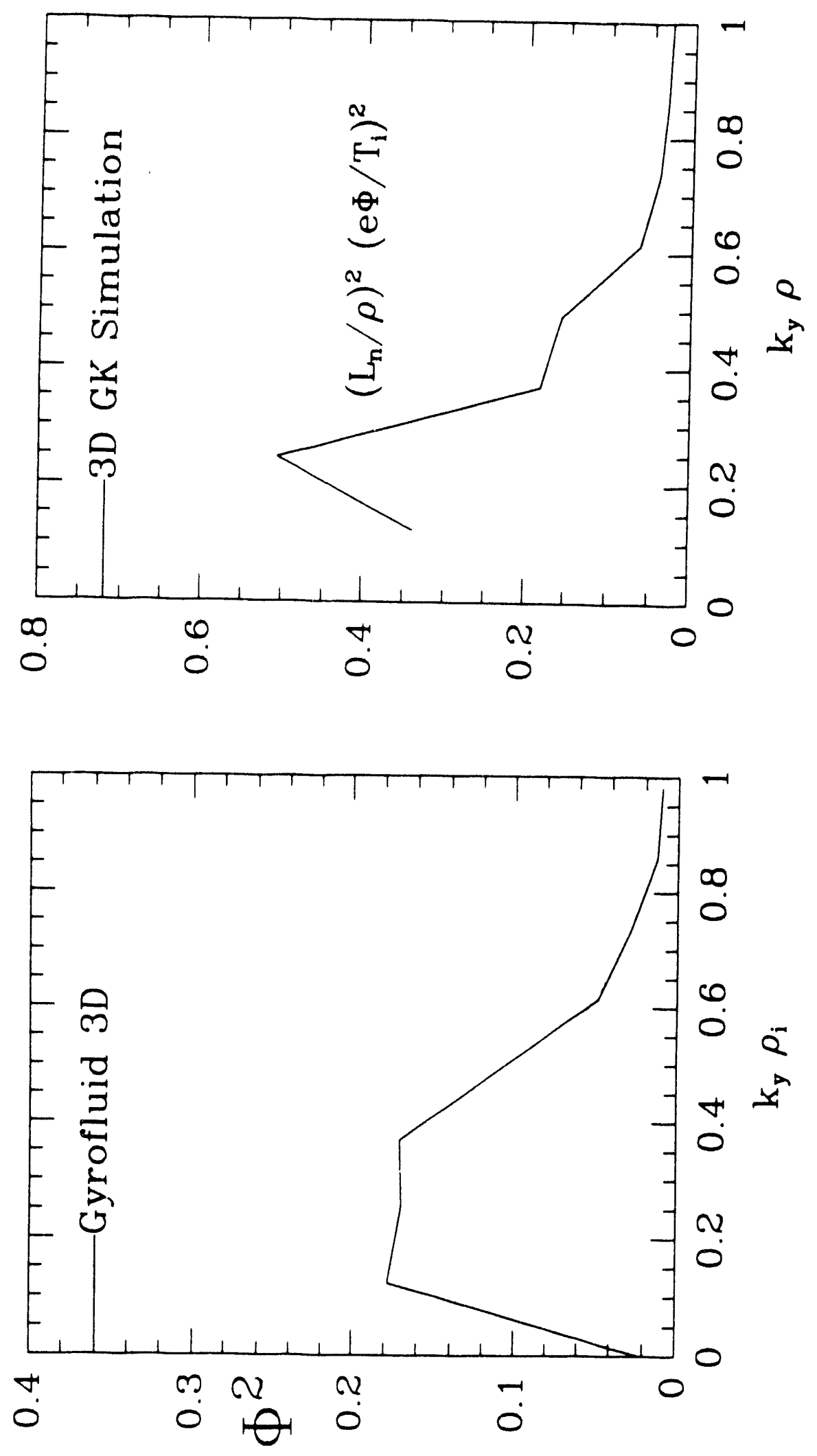

Figure 5 


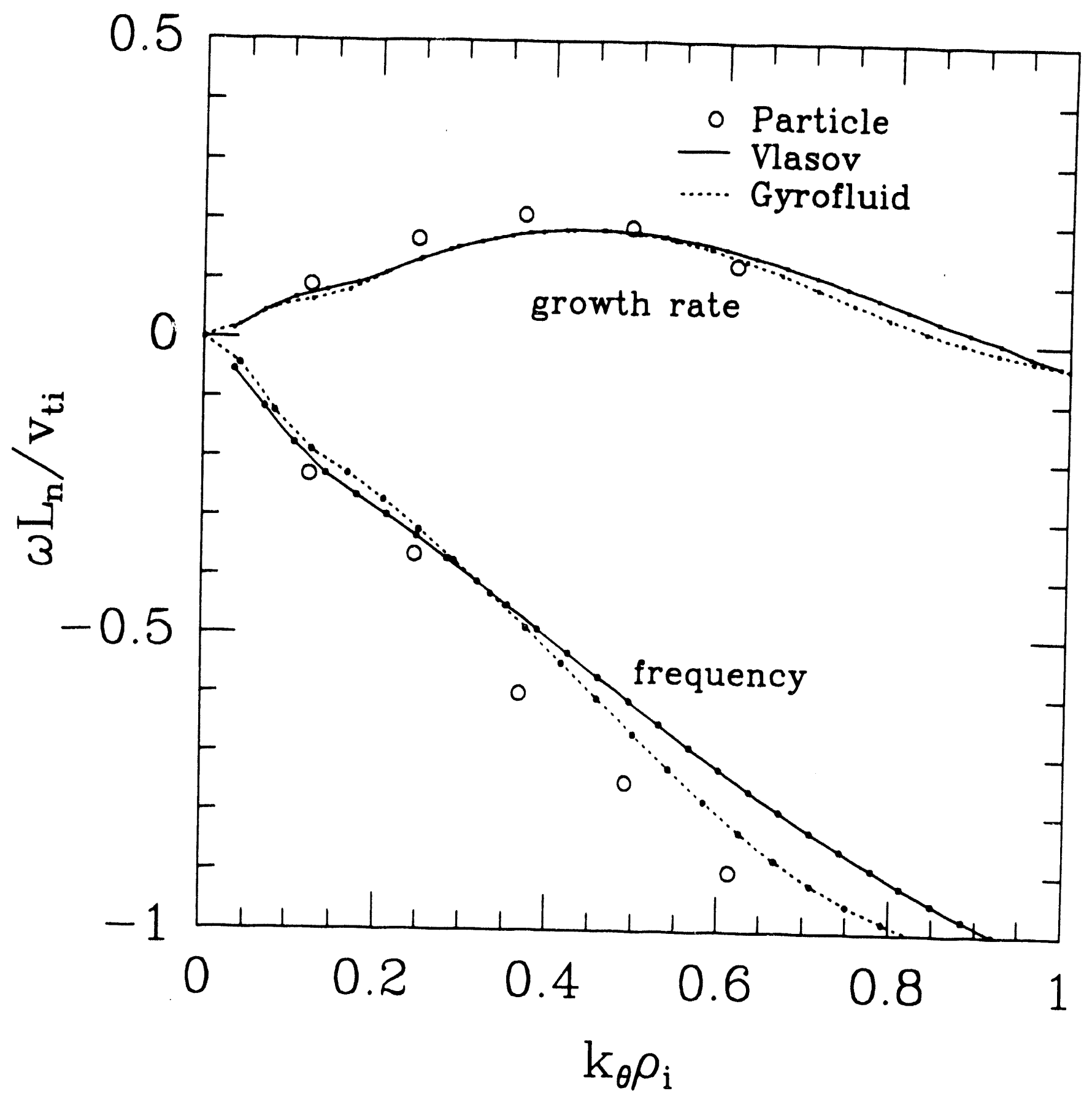

Figure 6 
Dr. F. Paoloni, Univ. of Wollongong, AUSTRALIA

Prof. M.H. Brennan, Univ, of Sydney, AUSTRALIA Plasma Research Lab., Australian Nat. Univ., AUSTRALIA

Prof. I.R. Jones, Flinders Univ, AUSTRALIA

Prol. F. Cap, Inst. for Theoretical Physics, AUSTRIA

Prol. M. Heindler, Institut fur Theoretische Physik, AUSTRIA

Prot. M. Goossens, Astronomisch Instituut, BELGIUM

Ecole Royale Militaire, Lab. de Phy. Plasmas, BELGIUM Commission-European, DG. XII-Fusion Prog., BELGIUM

Prof. R. Bouciqué, Rijksuniversiteit Gent, BELGIUM

Dr. P.H. Sakanaka, Instituto Fisica, BRAZIL

Prof. Dr. I.C. Nascimento, Instituto Fisica, Sao Paulo, BRAZIL Instituto Nacional De Pesquisas Espaciais-INPE, BRAZIL Documents Otfice, Atomic Energy of Canada Lid., CANADA Ms. M. Morin, CCFWTokamak de Varennes, CANADA Dr. M.P. Bachynski, MPB Technologies, Inc., CANADA Dr. H.M. Skarsgard, Univ. of Saskatchewan, CANADA Prof. J. Teichmann, Univ. of Montreal, CANADA

Prof. S.R. Sreenivasan, Univ. of Calgary, CANADA

Prof. T.W. Johnston, INRS-Energie, CANADA

Dr. R. Bolton, Centre canadien de fusion magnétique, CANADA

Dr. C.R. James., Univ. of Alberta, CANADA

Dr. P. Lukác, Komenského Universzita, CZECHO-SLOVAKIA

The Librarian, Culham Laboratory, ENGLAND

Library, R61, Ruthertord Appleton Laboratory, ENGLAND

Mrs. S.A. Hutchinson, JET Library. ENGLAND

Dr. S.C. Sharma, Univ. of South Pacific, FIJI ISLANDS

P. Mähönen, Univ. of Helsinki, FINLAND

Prof. M.N. Bussac, Ecole Polytechnique., FRANCE

C. Mouttet, Lab de Physique des Milieux lonisés, FRANCE

J. Radel, CEN/CADARACHE - Bat 506, FRANCE

Prot. E. Economou, Univ. of Crete, GREECE

Ms. C. Rinni, Univ. of loannina, GREECE

Preprint Library. Hungarian Academy of Sa., HUNGARY

Dr. B. DasGupta, Saha Inst. of Nuclear Physics, INDIA

Dr. P. Kaw, Inst. for Plasma Research, INDIA

Dr. P. Rosenau, Israel Inst. of Technology, ISRAEL

Librarian, Intemational Center for Theo Physics, ITALY

Miss C. De Palo, Associazione EURATOM-ENEA , ITALY

Dr. G. Grosso, Isotuto di Fisica del Plasma, ITALY

Prol. G. Rostangni, Istituto Gas lonizzati Del Cnr, ITALY
Dr. H. Yamato, Toshiba Res \& Devel Center, JAPAN

Prol. I. Kawakami, Hiroshima Univ., JAPAN

Prof. K. Nishikawa, Hiroshima Univ., JAPAN

Librarian, Naka Fusion Research Establishment, JAERI, JAPAN

Director, Japan Atomic Energy Research Inst. JAPAN

Prot. S. Itoh, Kyushu Univ., JAPAN

Research Info. Ctr., National Instit. for Fusion Science, JAPAN

Prof. S. Tanaka, Kyoto Univ., JAPAN

Library, Kyoto Univ., JAPAN

Prot. N. Inove, Univ. of Tokyo, JAPAN

Secretary, Plasma Section, Electrolechnical Lab., JAPAN

S. Mori, Technical Advisor, JAERI, JAPAN

Dr. O. Mitarai, Kumamoto Inst. of Technology, JAPAN

Dr. G.S. Lee. Korea Basic Sci. Ctr., KOREA

J. Hyeon-Sook, Korea Atomic Energy Research Inst., KOREA

D.I. Choi, The Korea Adv. Inst. of Sci. \& Tech., KOREA

Prol. B.S. Liley, Univ. of Waikato, NEW ZEALAND

Inst of Physics, Chinese Acad Sci PEOPLE'S REP. OF CHINA

Library, Inst. of Plasma Physics, PEOPLE'S REP. OF CHINA

Tsinghua Univ. Library, PEOPLE'S REPUBLIC OF CHINA

2. Li, S.W. Inst Physics, PEOPLE'S REPUBLIC OF CHINA

Prof. J.A.C. Cabral, Instituto Superior Tecnico, PORTUGAL

Prof. M.A. Hellberg, Univ of Natal, S. AFRICA

Prot. D.E. Kim, Pohang Inst. of Sci. \& Tech., SO. KOREA

Prot. C.I.E.M.A.T. Fusion Division Library, SPAIN

Dr. L. Stenflo, Univ. of UMEA, SWEDEN

Library, Royal Inst. of Technology, SWEDEN

Prot. H. Withelmson, Chalmers Univ. of Tech., SWEDEN

Centre Phys. Des Plasmas, Ecole Polytech, SWITZERLAND

Bibliotheek, Inst. Voor Plasma-Fysica, THE NETHERLANDS

Asst. Prol. Dr. S. Cakir, Middle East Tech. Univ., TURKEY

Dr. V.A. Glukhikh,Sci. Res. Inst. Electrophys.I Apparatus, USSR

Dr. D.D. Ryulov, Siberian Branch of Academy of Sci., USSR

Dr. G.A. Eliseev, I.V. Kurchatov Inst., USSR

Libranian, The Ukr.SSR Academy of Sciences, USSR

Dr. L.M. Kovizhnykh, Inst. of General Physics, USSR

Kemforschungsanlage GmbH, Zentralbibliothek, W. GERMANY

Bibliothek, Inst. Für Plasmatorschung, W. GERMANY

Prof. K. Schindler, Ruhr-Universitát Bochum, W. GERMANY

Dr. F. Wagner, (ASDEX), Max-Planck-Institut, W. GERMANY

Librarian, Max-Planck-Institut, W. GERMANY 
